\title{
An Adaptive Near-far Resistant Single-user Channel Estimation Algorithm for Multipath Fading DS-CDMA Systems
}

\author{
Ahmet Rizaner, Hasan Amca, Kadri Hacıoğlu ${ }^{1}$, Ali H. Ulusoy and Ansgar Scherb ${ }^{2}$ \\ Dep. of Electrical and Electronic Eng. \\ Eastern Mediterranean University \\ Magosa - Via Mersin 10 TURKEY \\ ${ }^{1}$ University of Colorado at Boulder \\ Center For Spoken Language \\ Research Boulder, CO80309 - USA \\ ${ }^{2}$ University of Bremen - FB1, Dept. of \\ Communications Engineering P.O.Box 3304 \\ 40, D-28334 Bremen, GERMANY
}

ahmet.rizaner@emu.edu.tr

\begin{abstract}
Channel estimation techniques for CDMA systems need to combat multiple access interference and near-far effects to improve the estimation performance. Linear single-user MMSE detector has certain advantages with respect to near-far problem. In this paper, single-user MMSE detector is used to develop an iterative near-far resistant radio channel estimation algorithm. Computer simulation results demonstrate that a significant performance improvement against conventional singleuser estimator can be achieved with the developed method especially under extreme near-far conditions.
\end{abstract}

\section{Introduction}

Reliable coherent communication over mobile wireless channels requires accurate estimation of multipath fading channel parameters. Multiple access interference (MAI) and near-far resistance are the main factors affecting the performance of channel estimation techniques for direct sequence code division multiple access (DS-CDMA) systems. Although, several multiuser channel estimation algorithms have been proposed to mitigate MAI, these algorithms require high computational complexities [1], [2]. In addition, the knowledge of the spreading sequences and timings of all the users are required. A multi-user near-far resistant channel estimation problem is addressed in [2] by using a linear decorrelating detector to remove the MAI prior to channel estimation. The techniques developed for singleuser communications, are mostly devoted to combat Gaussian white noise. However, even a small amount of near-far effect can drastically degrade the performance of single-user estimators and strict power control is necessary for reliable estimation. The effects of the nearfar problem on the performance of the single-user channel estimation systems have not yet been fully investigated, and practical solutions to the near-far resistant estimation problem is still to be found. A singleuser near-far resistant channel estimation problem is considered in [3] where interfering users are modeled as colored non-Gaussian noise. In this approach, a maximum-likelihood estimation technique is used that requires the knowledge of the transmitted symbols and suppresses the MAI by a whitening filter derived from the sample covariance matrix. However, the channels were restricted to be single path and not evaluated for multipath fading channels. Although, the estimation scheme developed in [3] exhibits the desired near-far resistance that the conventional single user approach fails to provide, there is a significant increase in the computational complexity. In this paper, we developed a method for near-far resistant channel estimation of a single-user in a multi-user environment using training sequences and approximate it by using an iterative algorithm to reduce the computational complexity in each symbol duration. The single-user linear minimum mean square (MMSE) detector has not been considered for channel estimation problem. The channel estimation approach is based on the linear single-user MMSE detector, which has certain advantages with respect to the near far problem [4]. The presented method in this paper assumes the knowledge of the desired user's spreading sequence only and is suitable for use in, for instance, slotted system where each user transmits a data burst with a training sequence. Simulation results show that the proposed method well mitigates the multi-user interference and it can be used to improve the channel estimation accuracy without requiring any additional information than standard single-user estimator. 


\section{System Model}

We considered a $P$-user synchronous CDMA system where direct paths from all users are perfectly synchronized. The proposed technique can easily be extended to the asynchronous case. The baseband signal plus additive white Gaussian noise $n(\mathrm{t})$ at the receiver is given by,

$$
r(t)=\sum_{i=1}^{P} A_{i} \sum_{n=1}^{N} b_{i}(n) \sum_{l=1}^{L} h_{i, l} c_{i}\left(t-(l-1) T_{c}-(n-1) T_{s}\right)+n(t)
$$

where $A_{i}$ is the transmitted amplitude of the ith user, $\left\{b_{i}(n) \in \pm 1\right\}$ is the information-bearing symbol, $\left\{C_{i}(j) \in \pm 1, j=1 \cdots L_{c}\right\}$ is the pre-assigned spreading code of the $i$-th user, $T_{c}$ is the chip period of spreading code waveforms, $T_{s}$ is the symbol duration, $L$ is the total number of propagation paths, each spaced at $T_{c}$ time intervals, of the channel, $h_{i, l}$ is the complex attenuation of the $l$-th propagation path and $N$ is the length of the frame containing $N_{p}$ training symbols. The channel is assumed to vary slowly such that the channel parameters are constant over the training period and changed independently from frame to frame.

By sampling the received signal $y(t)$ at chip rate over the training period, the received signal vector $\mathbf{y}$ of length $N_{p} L_{c}$ can be obtained as follows:

$$
\mathbf{y}=\mathbf{C h}+\mathbf{n}
$$

where $\mathbf{n}$ is the noise vector, $\mathbf{C}=\left[\begin{array}{lllll}\mathbf{C}(0) & \mathbf{C}(1) & \cdots & \mathbf{C}\left(N_{p}-1\right)\end{array}\right]^{T}$ is spread training sequences matrix, $\mathbf{h}=\left[\begin{array}{lllll}\mathbf{h}_{1} & \mathbf{h}_{2} & \cdots & \mathbf{h}_{P}\end{array}\right]^{T}$ is the channel coefficients vector in which the amplitudes, $A_{i}$, are accounted for as $\mathbf{h}_{i}=A_{i}\left[\begin{array}{llll}h_{i, 1} & h_{i, 2} & \cdots & h_{i, L}\end{array}\right]$, and $(\cdot)^{T}$ denotes the transpose operation. The $L_{c} \times L$ Toeplitz matrix of spreading sequence of the $i$-th user at the $n$-th bit interval can be represented as, $\mathbf{C}_{i}=\mathbf{C}_{i}^{R}+\mathbf{C}_{i}^{L}$, where, $\mathbf{C}_{i}^{R}$ is constructed with the right part of the spreading code of the $i$-th user corresponding to the current symbol and $\mathbf{C}_{i}^{L}$ is constructed using the left part of the spreading code of the $i$-th user corresponding to the previous symbol. These matrices can be defined as,

$$
\mathbf{C}^{R}=\left[\begin{array}{cccc}
c_{i}(1) & 0 & \cdots & 0 \\
c_{i}(2) & c_{i}(1) & \ddots & \ddots \\
\vdots & \vdots & \ddots & \ddots \\
C_{i}\left(L_{c}\right) & c_{i}\left(L_{c}-1\right) & \cdots & c_{i}\left(L_{c}-L+1\right)
\end{array}\right]
$$

and,

$$
\mathbf{C}_{i}^{L}=\left[\begin{array}{cccc}
0 & C_{i}\left(L_{c}\right) & \cdots & C_{i}\left(L_{c}-L+2\right) \\
0 & 0 & \ddots & \vdots \\
\vdots & \vdots & \ddots & C_{i}\left(L_{c}\right) \\
0 & 0 & \cdots & 0
\end{array}\right]
$$

Hence, the $L_{c} \times P L$ spread training sequences matrix at the $n$-th symbol interval can be constructed as,

$$
\mathbf{C}(n)=\left[b_{1}(n-1) \mathbf{C}_{1}^{L}+b_{1}(n) \mathbf{C}_{1}^{R} \quad \cdots \quad b_{p}(n-1) \mathbf{C}_{p}^{L}+b_{p}(n) \mathbf{C}_{p}^{R}\right]
$$

\section{Channel Estimation}

The linear MMSE detector, that has certain advantages with respect to the near-far problem, is used to obtain the channel coefficients. The standard singleuser MMSE detector is defined by [4],

$$
\mathbf{v}_{i}=\mathbf{R}^{-1} \mathbf{C}_{i} \mathbf{h}_{i}
$$

where, $\mathbf{R}$ is $L_{c} \times L_{c}$ data covariance matrix that can be estimated by temporal averaging as,

$$
\mathbf{R}=\frac{1}{N} \sum_{n=1}^{N} \mathbf{y}(n) \mathbf{y}^{H}(n)
$$

here $\mathbf{y}(n)$ is the discrete vector of the received samples at the $n$-th symbol interval and $(\cdot)^{H}$ denotes the conjugate transpose operation. The target function for the channel estimation implying single-user MMSE detection for the transmitted sequence can be defined as,

$$
\begin{aligned}
f\left(\mathbf{h}_{i}\right) & =E\left\{\left\|\mathbf{v}_{i}^{H} \mathbf{X}-\mathbf{b}_{i}^{T}\right\|^{2}\right\} \\
& =E\left\{\left\|\mathbf{h}_{i}^{H} \mathbf{C}_{i}^{H} \mathbf{R}^{-1} \mathbf{X}-\mathbf{b}_{i}^{T}\right\|^{2}\right\}
\end{aligned}
$$

where, $\mathbf{X}=\left[\begin{array}{llll}\mathbf{y}(1) & \mathbf{y}(2) & \cdots & \mathbf{y}(N)\end{array}\right]$ is the received samples matrix and $\mathbf{b}_{i}=\left[\begin{array}{lllll}b_{i}(1) & b_{i}(2) & \cdots & b_{i}(N)\end{array}\right]^{T}$ is the vector of transmitted data sequence of the $i$-th user. The closed-form solution of the channel impulse response 
(CIR) satisfying $\hat{\mathbf{h}}_{i}=\arg \min f\left(\mathbf{h}_{i}\right)$ is obtained by setting the partial derivative $\partial f\left(\mathbf{h}_{i}\right) / \partial \mathbf{h}_{i}^{H}$ to zero as,

$$
\frac{d f\left(\mathbf{h}_{i}\right)}{d \mathbf{h}_{i}^{H}}=\mathbf{C}_{i}^{H} \mathbf{R}^{-1} \mathbf{C}_{i} \hat{\mathbf{h}}_{i}-\mathbf{C}_{i}^{H} \mathbf{R}^{-1} \mathbf{X} \mathbf{b}_{i}=0
$$

that implies that,

$$
\hat{\mathbf{h}}_{i}=\left(\mathbf{C}_{i}^{H} \mathbf{R}^{-1} \mathbf{C}_{i}\right)^{-1} \mathbf{C}_{i}^{H} \mathbf{R}^{-1} \mathbf{X} \mathbf{b}_{i}
$$

In order to simplify the channel response estimation, (7) can be transformed into an iterative form as,

$$
\begin{aligned}
\hat{\mathbf{h}}_{i}(n+1) & =\hat{\mathbf{h}}_{i}(n)+\left.\mu \frac{d f\left(\mathbf{h}_{i}\right)}{d \mathbf{h}_{i}^{H}}\right|_{\mathbf{h}_{i}=\hat{\mathbf{h}}_{i}(n)} \\
& =\hat{\mathbf{h}}_{i}(n)+\mu \mathbf{C}_{i}^{H} \mathbf{R}^{-1}\left(\mathbf{C}_{i} \hat{\mathbf{h}}_{i}(n)-\mathbf{X} \mathbf{b}_{i}\right)
\end{aligned}
$$

where $\mu$ is the step-size parameter that controls the rate of convergence of the algorithm. In (8), $\mathbf{b}_{i}$ and $\mathbf{X}$ extend over the whole data record. Starting from an initial guess $\hat{\mathbf{h}}_{i}(0)$, we can update the CIR estimate for each received training sequence recursively in time and spread the computation over training length to provide a reasonable complexity as follows,

$$
\hat{\mathbf{h}}_{i}(n+1)=\hat{\mathbf{h}}_{i}(n)+\mu \mathbf{C}_{i}^{H} \mathbf{R}^{-1}\left(\mathbf{C}_{i} \hat{\mathbf{h}}_{i}(n)-b_{i}(n) \mathbf{y}(n)\right)
$$

A large value of step-size $(\mu)$ would provide faster adaptation but could lead to divergence of the channel estimate. On the other hand, if $\mu$ is too small, we will lose a significant amount of data while trying to acquire the channel. The optimal step-size depends on the eigenvalues of the covariance matrix $\mathbf{R}$ [5]. Step-size can be chosen time varying and quite often the optimum stepsize is approximated as $\mu(n)=\mu_{o} /\left(\mathbf{y}^{H}(n) \mathbf{y}(n)\right) ; 0<\mu_{o}<1$ [6].

\section{Simulation Results}

In this section, the effectiveness of the proposed channel estimation method and its iterative implementation is illustrated by computer simulations and compared to the single-user channel estimate, which corresponds to a sliding correlator approach, given by,

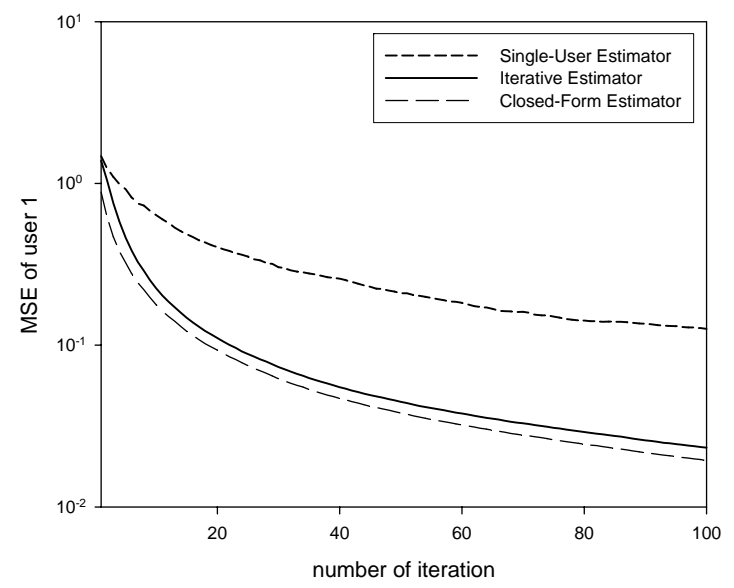

Fig. 1. Mean squared error of channel estimate for user 1 $\left(\mathrm{SNR}=8 \mathrm{~dB}, \mathrm{NFR}=10 \mathrm{~dB}, P=15, L=4, L_{c}=31\right)$

$$
\hat{\mathbf{h}}_{i}^{s u}=\frac{1}{N_{P}} \sum_{n=1}^{N_{p}} b_{i}(n) \mathbf{C}_{i}^{H} \mathbf{y}(n)
$$

We consider a 15-user synchronous CDMA system. The desired and interfering users employed Gold sequences of length $L_{c}=31$ as spreading codes. A fourpath slowly varying propagation channel is simulated for each user. Channels are randomly realized with exponential power delay profile with a dynamic range of $20 \mathrm{~dB}$. We assume that the channel coefficients are Gaussian distributed and their variances are determined by the exponential power profile and norm of the channel vector $\mathbf{h}_{i}$, is one. The preamble bits and data bits are randomly generated for each user as antipodal signaling. Performance measure of the channel estimation is the average mean squared error (MSE) of the channel estimates. The MSE of the channel parameter estimate has been calculated by averaging 200 Monte-Carlo runs. In each run, different channel parameters and training symbols are used.

Figure 1 shows the improvement in average mean squared error of the closed-form estimation and the iterative estimation algorithm against the single-user channel estimate with training length when the desired user has $10 \mathrm{~dB}$ less power that the interfering users. The adaptive step-size, $\mu(n)=0.02 / n$, that is initially large and decreasing for each channel update, is chosen to combine the desirable features of large and small stepsizes. The simulation result shows that, iterative channel estimation results in significant performance improvement 


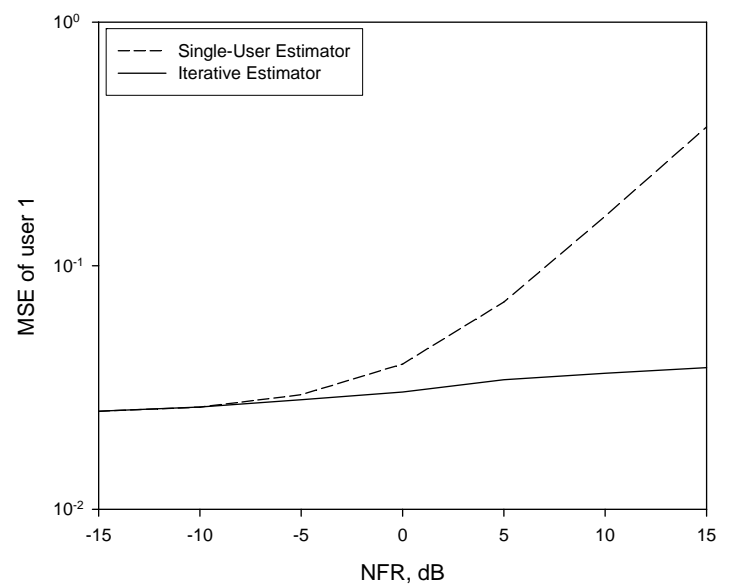

Fig. 2. MSE versus NFR for user $1\left(\mathrm{SNR}=8 \mathrm{~dB}, N_{P}=64\right.$, $\left.P=15, L=4, L_{c}=31\right)$

compared to the single-user estimator and it quite well approximates the closed-form solution.

Figure 2 depicts the near-far resistance of the proposed iterative and single-user estimator with 64 training symbols at $8 \mathrm{~dB}$ signal to noise ratio (SNR). The near-far ratio varied from $-15 \mathrm{~dB}$ to $15 \mathrm{~dB}$. We define the near-far ratio (NFR) as the ratio of the average received power $\left(A_{a v}^{2}\right)$ of each interferer to that of the desired user by $A_{a v}^{2} / A_{i}^{2}$, where the amplitude of the transmission for the interfering user $k$ is $A_{k}=1$ for all $k \neq i$. As evident from the simulation results, the developed iterative algorithm is near-far resistant, that is, its performance is independent of the interfering users' powers. It is also worth noting that this result shows significant gains even in the equal power case. When the interfering users have higher powers than the desired user, MAI can further degrade the single-user estimate. That is, single-user estimator is not near-far resistant and for strong MAI it fails to show satisfactory performance. Thus, power control is necessary with single-user estimator to improve CIR estimation performance of all the users.

It is well known that performance of single-user estimators are limited by MAI. However, the developed single-user channel estimator eliminates MAI and increase system capacity. In Figure 3, the effect of the MAI on the iterative estimator is investigated by increasing the system load (number of users). Curves are obtained by using 64 training symbols in each slot at 8 $\mathrm{dB}$ SNR. In this simulation, the desired user has $10 \mathrm{~dB}$ less power that the interfering users. Simulation result shows that, MAI is well mitigated by iterative algorithm. However, the performance of the single-user estimator is very sensitive to MAI.

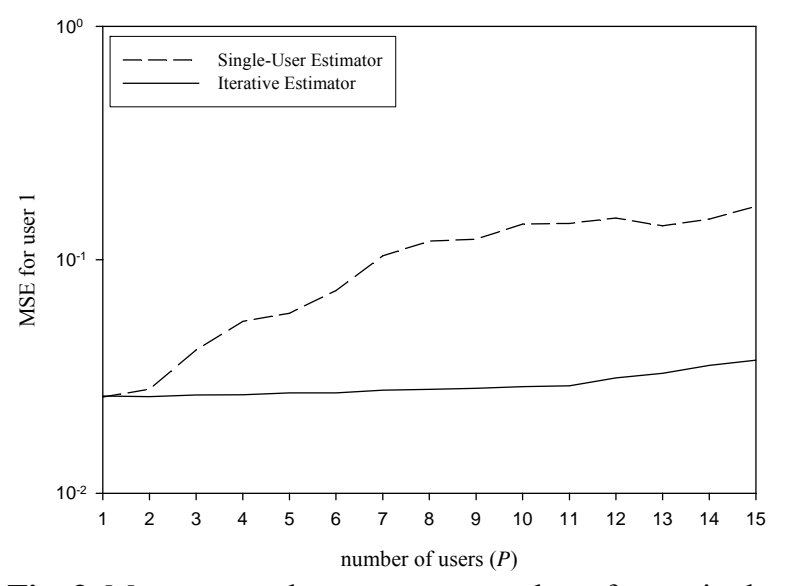

Fig. 3. Mean squared error versus number of users in the system $\left(\mathrm{SNR}=8 \mathrm{~dB}, \mathrm{NFR}=10 \mathrm{~dB}, N_{p}=64, L=4, L_{c}=31\right)$

\section{Conclusion}

In this paper, a single-user channel estimator based on single-user MMSE detection criteria is proposed for multipath fading DS-CDMA channels and iteratively implemented to reduce the computational complexity of the exact estimation. The proposed scheme improves the channel estimation accuracy of single-user estimator significantly specially under high near-far situations and does not require any additional information than standard single-user estimator. The estimated channel coefficients can be used to implement any detector requiring the knowledge of these coefficients for data detection phase.

\section{References}

[1] S.J. Grant and J.K. Cavers, "Multiuser channel estimation for detection of cochannel signals," IEEE Internat. Conf. on Commun. June 1999.

[2] M. Missiroli, Y. J. Guo and S. K. Barton, "Near-far resistant channel estimation for CDMA systems using the linear decorrelating detector", IEEE Trans. on Commun., vol. 48, no. 3, pp. 514-524, March 2000.

[3] S.E. Bensley and B. Aazhang, "Maximum-likelihood synchronization of a single user for code-division multiple-access communication systems", IEEE Trans. on Commun., vol. 46, no. 3, pp. 392-399, March 1998.

[4] S. Verdu, Multiuser Detection, Cambridge University Press, Cambridge, New York, 1998.

[5] S. Haykin, Adaptive Filter Theory, 2nd Ed., Englewood Cliffs, NJ: Prentice-Hall, 1991.

[6] M. Latva-aho and J. J. Juntti, "LMMSE detection for DSCDMA systems in fading channels", IEEE trans. commun., vol.48, no. 2, pp. 194-199, 2000. 